Pacific Journal of Mathematics

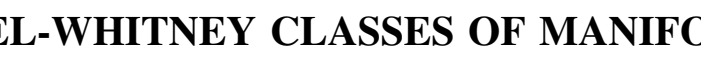




\title{
STIEFEL-WHITNEY CLASSES OF MANIFOLDS
}

\author{
R. E. STONG
}

1. Introduction. The purpose of this paper is to axiomatize the Stiefel-Whitney classes of closed manifolds and using the axioms to give a proof of Wu's theorem ([3]):

THEOREM. If $M^{n}$ is a closed $n$ manifold and $v=1+v_{1}+\cdots+v_{n}$ with $v_{i} \in H^{i}\left(M ; Z_{2}\right)$ defined by

$$
\left\langle v_{1} \cup x,[M]\right\rangle=\left\langle S q^{i} x,[M]\right\rangle
$$

for all $x \in H^{n-i}\left(M ; Z_{2}\right)$, then $w=S q v$ is the Stiefel-Whitney class of $M$.

The axioms for the Stiefel-Whitney classes are as follows.

For each closed manifold $M^{n}$ there is a class $\alpha(M)=$ $\alpha_{0}(M)+\cdots+\alpha_{n}(M)$ where $\alpha_{\imath}(M) \in H^{i}\left(M ; Z_{2}\right)$ satisfying:

(1) If $i: M \rightarrow N$ is an imbedding with trivial normal bundle, then $\alpha(M)=i^{*} \alpha(N)$,

(2) $\alpha(M \times N)=\pi_{M}^{*}(\alpha(M)) \cup \pi_{N}^{*}(\alpha(N))$, and

(3) $\alpha(R P(n))=(1+a)^{n+1}$, where $a \in H^{1}\left(R P(n) ; Z_{2}\right)$ is the nonzero class.

It is well-known (See [2]) that the Stiefel-Whitney classes satisfy these properties, and it will be shown that if $\alpha(M)$ satisfies these properties, then $\alpha(M)=w(M)$.

A different axiomatization has been given by J. D. Blanton and P. A. Schweitzer, "Axioms for characteristic classes of manifolds", Proc. of Symp. in Pure Math., Amer. Math. Soc. 27 (1975), volume I, 349-356. Their axioms use all manifolds, so cannot be used to prove Wu's theorem which is only meaningful for closed manifolds.

2. Axiomatics. Suppose one is given classes $\alpha(M) \in$ $H^{*}\left(M ; Z_{2}\right)$ for each closed manifold $M^{n}$ which satisfy properties 1,2 , and 3 .

LEMMA 1. $\alpha_{0}(M)=1$, i.e., is the unit class.

Proof. If $P$ is a point and $i: P \rightarrow R P(n)$ then $i$ is an imbedding with trivial normal bundle so $\alpha_{0}(P)=1$. Then for any $f: P \rightarrow M, f$ is an imbedding with trivial normal bundle, so $f^{*} \alpha_{0}(M)=1$. Thus $\alpha_{0}(M)=$ $1 .^{*}$ 
LEMMA 2. $\alpha\left(S^{n}\right)=1$.

Proof. For $n=0$ this is $\alpha_{0}\left(S^{0}\right)=1$. If $n>0, S^{n}$ imbeds in $S^{n+1}$ with trivial normal bundle, so $\alpha\left(S^{n}\right)=i^{*}\left(1+\alpha_{n+1}\left(S^{n+1}\right)\right)=1$. $^{*}$

Now consider a smooth vector bundle $\eta$ over a closed manifold $M$ and let $S=S(\eta \oplus 1)$ be the unit sphere bundle of the Whitney sum of $\eta$ and a trivial line bundle. Considering $S \subset E(\eta) \times R$ as the pairs $(x, t)$ with $\|x\|^{2}+t^{2}=1$, let $\sigma: M \rightarrow S$ by $\sigma(y)=(0 y, 1)$ where $0 y$ is the zero vector over $y$. Then $\sigma: M \rightarrow S$ is an imbedding with normal bundle $\eta$.

Define $\alpha(\eta)=\sigma^{*}(\alpha(S)) / \alpha(M)$.

Note. $\alpha(M)$ is invertible since $\alpha_{0}(M)=1$, and $\alpha_{0}(\eta)=1$.

LEMMA 3. If $f: M \rightarrow N$ is an imbedding with normal bundle $\nu$, then $\alpha(\nu)=f^{*}(\alpha(N)) / \alpha(M)$.

Proof. Let $g: M \rightarrow N \times S^{1}$ by $g(m)=(f(m), 1)$. Then $g$ is an imbedding with normal bundle $\nu \oplus 1$ and extends to an imbedding

$$
h: D(\nu \oplus 1) \rightarrow N \times S^{1}
$$

of the disc bundle. Clearly $g$ is homotopic to $h \circ \sigma, \sigma: M \rightarrow S(\nu \oplus 1)$ and $h: S(\nu \oplus 1) \rightarrow N \times S^{1}$ is an imbedding with trivial normal bundle. Now

$$
\begin{aligned}
\alpha(\nu) & =\frac{\sigma^{*} \alpha(S)}{\alpha(M)}=\frac{\sigma^{*} h^{*} \alpha\left(N \times S^{1}\right)}{\alpha(M)}=\frac{g^{*} \alpha\left(N \times S^{1}\right)}{\alpha(M)} \\
& =\frac{g^{*}\left(\pi_{N}^{*}(\alpha(N)) \cup \pi_{S}^{*} 1\left(\alpha\left(S^{1}\right)\right)\right)}{\alpha(M)}=\frac{g^{*} \pi_{N}^{*}(\alpha(N))}{\alpha(M)}=\frac{f^{*}(\alpha(N))}{\alpha(M)} .^{*}
\end{aligned}
$$

COROllary 1. If $f: M \rightarrow N$ is a smooth map $f^{*}(\alpha(N))=\alpha\left(f^{*} \tau_{N}\right)$, where $\tau_{N}$ is the tangent bundle of $N$.

Proof. $g: M \rightarrow M \times N$ given by $g(m)=(m, f(n))$ is an imbedding with normal bundle $f^{*} \tau_{N}$. Thus

$$
\begin{aligned}
\alpha\left(f^{*} \tau_{N}\right) & =g^{*}(\alpha(M \times N)) / \alpha(M)=g^{*}\left(\pi_{M}^{*}(\alpha(M)) \cup \pi_{N}^{*}(\alpha(N))\right) / \alpha(M) \\
& =\alpha(M) \cup f^{*}(\alpha(N)) / \alpha(M)=f^{*}(\alpha(N)){ }^{*}
\end{aligned}
$$

Corollary 2. $\alpha(M)=\alpha\left(\tau_{M}\right)$.

Proof. Apply Corollary 1 to the identity map.* 
CoRollary 3. If $\rho$ is a bundle over $M, \alpha\left(\rho \oplus \tau_{M}\right)=\alpha(\rho) \cup \alpha\left(\tau_{M}\right)$.

Proof. $\quad \sigma: M \rightarrow S=S(\rho \oplus 1)$ is an imbedding with normal bundle $\rho$ so $\alpha(\rho)=\sigma^{*} \alpha(S) / \alpha(M)$ or $\alpha(\rho) \cdot \alpha\left(\tau_{M}\right)=\sigma^{*}(\alpha(S))=\alpha\left(\sigma^{*} \tau_{S}\right)=$ $\alpha\left(\rho \oplus \tau_{M}\right){ }^{*}$

LEMMA 4. If $\xi$ and $\eta$ are smooth vector bundles over $M, \alpha(\xi \oplus \eta)=$ $\alpha(\xi) \cup \alpha(\eta)$.

Proof. Consider the composite

$$
f: M \stackrel{\Delta}{\rightarrow} M \times M \stackrel{\sigma_{1} \times \sigma_{2}}{\longrightarrow} S(\xi \oplus 1) \times S(\eta \oplus 1)=X .
$$

This is an imbedding with normal bundle $\xi \oplus \eta \oplus \tau_{M}$ so $f^{*}(\alpha(X))=$ $\alpha\left(\xi \oplus \eta \oplus \tau_{M}\right) \cup \alpha(M)=\alpha(\xi \oplus \eta) \cup \alpha(M) \cup \alpha(M)$. On the other hand,

$$
\begin{aligned}
f^{*}(\alpha(X)) & =f^{*}\left(\pi_{1}^{*}(\alpha(S(\xi \oplus 1))) \cup \pi_{2}^{*}(\alpha(S(\eta \oplus 1)))\right) \\
& =f^{*} \pi_{1}^{*}(\alpha(S(\xi \oplus 1))) \cup f^{*} \pi_{2}^{*}(\alpha(S(\eta \oplus 1))) \\
& =\sigma_{1}^{*}(\alpha(S(\xi \oplus 1))) \cup \sigma_{2}^{*}(\alpha(S(\eta \oplus 1))) \\
& =\alpha(\xi) \cup \alpha(M) \cup \alpha(\eta) \cup \alpha(M) .{ }^{*}
\end{aligned}
$$

LEMMA 5. If $\rho$ is a smooth vector bundle over $N$ and $f: M \rightarrow N$ is a smooth map, then $f^{*}(\alpha(\rho))=\alpha\left(f^{*} \rho\right)$.

Proof. Let $g: M \rightarrow S=S(\rho \oplus 1)$ be the composite of $f: M \rightarrow N$ and $\sigma: N \rightarrow S$. Then

$$
\begin{aligned}
g^{*}(\alpha(S)) & =f^{*} \sigma^{*}(\alpha(S))=f^{*}(\alpha(N) \cup \alpha(\rho)), \\
& =f^{*}(\alpha(N)) \cup f^{*}(\alpha(\rho))
\end{aligned}
$$

and

$$
\begin{aligned}
g^{*}(\alpha(S)) & =g^{*}\left(\alpha\left(\tau_{S}\right)\right)=\alpha\left(g^{*} \tau_{S}\right)=\alpha\left(f^{*} \sigma^{*} \tau_{S}\right) \\
& =\alpha\left(f^{*}\left(\tau_{N} \oplus \rho\right)\right)=\alpha\left(f^{*} \tau_{N} \oplus f^{*} \rho\right) \\
& =\alpha\left(f^{*} \tau_{N}\right) \cup \alpha\left(f^{*} \rho\right) .
\end{aligned}
$$

Since $f^{*}(\alpha(N))=\alpha\left(f^{*} \tau_{N}\right)$, the result follows. ${ }^{*}$

LEMMA 6. If $\xi$ is the nontrivial line bundle over $R P(n)$, then $\alpha(\xi)=1+a$. 
Proof. If $i: R P(n) \rightarrow R P(n+1)$ is the standard inclusion, then $i$ is an imbedding with normal bundle $\xi$, so $\alpha(\xi)=i^{*}(1+a)^{n+2} /(1+a)^{n+1}=$ $1+a .^{*}$

Proposition 1. $\alpha(M)$ is the Stiefel-Whitney class of the manifold $M$.

Proof. From Lemmas 4, 5, and 6, $\alpha(\eta)$ is the Stiefel-Whitney class of the bundle $\eta$, for these axioms characterize the Stiefel-Whitney classes of smooth bundles. From Corollary 2, one has $\alpha(M)=\alpha\left(\tau_{M}\right)=$ $w\left(\tau_{M}\right)^{*}$

3. Wu's theorem. For a closed $n$-manifold $M^{n}$, let $v(M)=$ $v_{0}(M)+\cdots+v_{n}(M) \in H^{*}\left(M ; Z_{2}\right)$ be the classes defined by

$$
\left\langle v_{i}(M) \cup x,[M]\right\rangle=\left\langle S q^{i} x,[M]\right\rangle
$$

for all $x \in H^{n-1}\left(M ; Z_{2}\right)$. Let $\alpha(M)=S q(v(M))$.

LEMMA 7. If $i: M \rightarrow N$ is an imbedding with trivial normal bundle, then $i^{*}(v(N))=v(M)$, and $i^{*}(\alpha(N))=\alpha(M)$.

Proof. If $m=\operatorname{dim} M, n=\operatorname{dim} N$, let

$$
i_{*}: H^{\prime}\left(M ; Z_{2}\right) \rightarrow H^{H^{+n-m}}\left(N ; Z_{2}\right)
$$

be the Gysin homomorphism defined by

$$
\left\langle i_{*}(x) \cup y,[N]\right\rangle=\left\langle x \cup i^{*}(y),[M]\right\rangle
$$

for all $x \in H^{\prime}\left(M ; Z_{2}\right)$ and $y \in H^{m-j}\left(N ; Z_{2}\right)$. Let $i$ be extended to an imbedding

$$
j: M \times D^{n-m} \rightarrow N
$$

and let

$$
c: N \rightarrow M \times D^{n-m} / M \times S^{n-m-1}
$$

be the collapse map sending all points not in the interior of the image of $j$ to the base point. Identify $\tilde{H}^{*}\left(M \times D^{n-m} / M \times S^{n-m-1} ; Z_{2}\right)$ with $H^{*}\left(M ; Z_{2}\right) \otimes \tilde{H}^{*}\left(D^{n-m}, S^{n-m-1} ; Z_{2}\right)$ and then

$$
i_{*}(x)=c^{*}(x \otimes \sigma)
$$

where $\sigma \in H^{n-m}\left(D^{n-m}, S^{n-m-1} ; Z_{2}\right)$ is the nonzero class. 
Note. To see this $c_{*}$ sends the fundamental class of $N$ to $[M] \otimes$ (dual $\sigma$ ) for it is degree one on top dimensional simplices. Letting $V=N$-interior $\quad\left(j\left(M \times D^{n-m}\right)\right), \quad$ and $\quad W=M \times D^{n-m}, \quad(N, V) \cong$ $\left(M \times D^{n-m}, M \times S^{n-m-1}\right)$ by excision, and the module structure of $H^{*}\left(N, V ; Z_{2}\right)$ as $H^{*}\left(N ; Z_{2}\right)$ module factors through $H^{*}\left(j W ; Z_{2}\right)$, i.e.

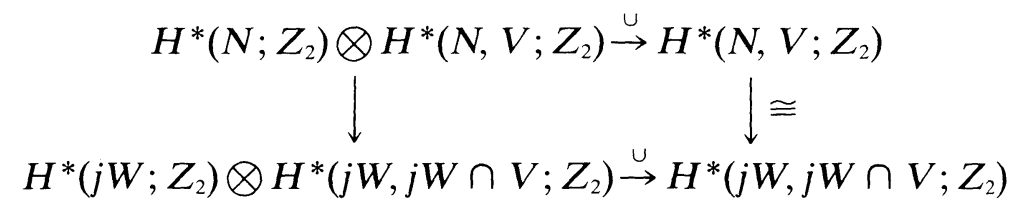

commutes. Thus $y \cup c^{*}(x \otimes \sigma)=c^{*}\left(i^{*}(y) \cup x \otimes \sigma\right)$. (See [1]).

Then

$$
\begin{aligned}
\left\langle i^{*}\left(v_{l}\right) \cup x,[M]\right\rangle & =\left\langle v_{\jmath} \cup i_{*}(x),[N]\right\rangle \\
& =\left\langle S q^{\prime} i_{*}(x),[N]\right\rangle \\
& =\left\langle S q^{\prime} c^{*}(x \otimes \sigma),[N]\right\rangle \\
& =\left\langle c^{*}\left(S q^{\prime}(x \otimes \sigma)\right),[N]\right\rangle \\
& =\left\langle c^{*}\left(\left(S q^{j} x\right) \otimes \sigma\right),[N]\right\rangle \\
& =\left\langle i_{*}\left(S q^{j} x\right) \cup 1,[N]\right\rangle \\
& =\left\langle S q^{\prime} x,[M]\right\rangle \\
& =\left\langle v_{j} \cup x,[M]\right\rangle
\end{aligned}
$$

for all $x$, so $i^{*}\left(v_{l}(N)\right)=v_{l}(M)$. Squaring gives $i^{*} \alpha(N)=i^{*} \operatorname{Sqv}(N)=$ $S q i^{*} v(N)=S q v(M)=\alpha(M){ }^{*}$

LEMMA 8. $\quad v(M \times N)=\pi_{M}^{*} v(M) \cup \pi_{N}^{*} v(N)$ and $\quad \alpha(M \times N)=$ $\pi_{M}^{*} \alpha(M) \cup \pi_{N}^{*} \alpha(N)$.

Proof. Identify $H^{*}\left(M \times N ; Z_{2}\right)$ with $H^{*}\left(M ; Z_{2}\right) \otimes H^{*}\left(N ; Z_{2}\right)$ so that

$$
\left\langle\pi_{M}^{*}(a) \cdot \pi_{N}^{*}(b),[M \times N]\right\rangle=\langle a,[M]\rangle \cdot\langle b,[N]\rangle,
$$

and the result follows easily from the axioms for $S q^{i}{ }^{*}$

LEMMA 9. $\quad v(R P(n))=\sum_{i=0}^{n}\left(\begin{array}{c}n-i \\ i\end{array}\right) a^{i}$ and $\alpha(R P(n))=(1+a)^{n+1}$. 
Proof. Since $\quad S q^{i} a^{n-\imath}=\left(\begin{array}{c}n-i \\ i\end{array}\right) a^{n}, \quad v_{1}=\left(\begin{array}{c}n-i \\ i\end{array}\right) a^{i}$. In $H^{*}\left(R P(\infty) ; Z_{2}\right)$ define classes

$$
v(n)=\sum_{i=0}^{n}\left(\begin{array}{c}
n-i \\
i
\end{array}\right) a^{i}
$$

Then

$$
\begin{aligned}
v(n+1) & =\sum_{i=0}^{n+1}\left(\begin{array}{c}
n+1-i \\
i
\end{array}\right) a^{i} \\
& =\sum_{i=0}^{n+1}\left\{\left(\begin{array}{c}
n-i \\
i
\end{array}\right)+\left(\begin{array}{c}
n-i \\
i-1
\end{array}\right)\right\} a^{i} \\
& =\sum_{i=0}^{n+1}\left\{\left(\begin{array}{c}
n-i \\
i
\end{array}\right)+\left(\begin{array}{c}
n-1-(i-1) \\
i-1
\end{array}\right)\right\} a^{i} \\
& =v(n)+a v(n-1),
\end{aligned}
$$

with $v(0)=v(1)=1 . \quad$ Letting $\alpha(n)=S q v(n)$,

$$
\begin{aligned}
\alpha(n+1) & =S q(v(n)+a v(n-1)) \\
& =\alpha(n)+\left(a+a^{2}\right) \alpha(n-1)
\end{aligned}
$$

with $\alpha(0)=\alpha(1)=1$.

Then it is easy to see $\alpha(n)=(1+a)^{n+1}-a^{n+1}$ by induction. Since $\alpha(R P(n))$ is the pull back of $\alpha(n)$ and $a^{n+1}=0$, the result follows. ${ }^{*}$

From Proposition 1 , one then has $\alpha(M)=w(M)$ for all $M$, which is Wu's theorem.

\section{REFERENCES}

1. M. F. Ativah and F. Hirzebruch, Cohomologie Operationen und charakteristische Klassen, Math. Zeit., 77 (1961), 149-187.

2. J. W. Milnor, Lectures on characteristıc classes, mimeographed, Prınceton Unıversity, Prınceton, N J., 1957.

3. Wen-Tsün Wu, Classes caractéristiques et l-carrés d'une variété, C. R Acad. Sci. Paris, 230 (1950), 508-511.

Recerved July 13, 1976. The author is indebted to the Natronal Science Foundation for financial support during this work. 



\section{Pacific Journal of Mathematics}

Vol. 68, No. 1

March, 1977

Richard Julian Bagby, On $L^{p}, L^{q}$ multipliers of Fourier transforms . .......

Robert Beauwens and Jean-Jacques Van Binnebeek, Convergence theorems in

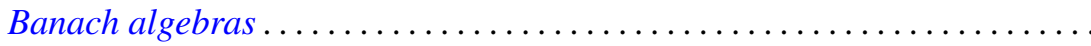

James Cyril Becker, Skew linear vector fields on spheres in the stable

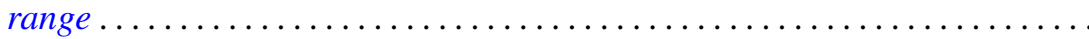

Michael James Beeson, Continuity and comprehension in intuitionistic formal

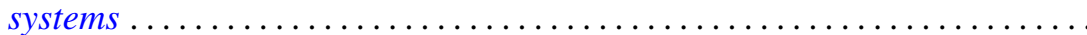

James K. Deveney, Generalized primitive elements for transcendental field

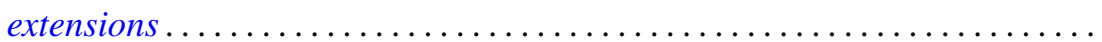

Samuel S. Feder, Samuel Carlos Gitler and K. Y. Lam, Composition properties

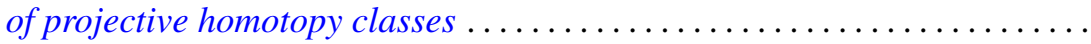

Nathan Jacob Fine, Tensor products of function rings under composition ......

Benno Fuchssteiner, Iterations and fixpoints . . . . . . . . . . . . . .

Wolfgang H. Heil, On punctured balls in manifolds

Shigeru Itoh, A random fixed point theorem for a multivalued contraction

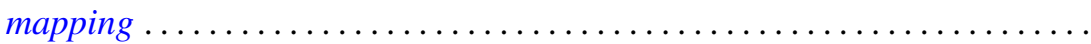

Nicolas P. Jewell, Continuity of module and higher derivations . . . . . . ......

Roger Dale Konyndyk, Residually central wreath products . . . . . . . . . . .

Linda M. Lesniak and John A. Roberts, On Ramsey theory and graphical

parameters.

Vo Thanh Liem, Some cellular subsets of the spheres.

Dieter Lutz, A perturbation theorem for spectral operators

P. H. Maserick, Moments of measures on convex bodies ... . . .

Stephen Joseph McAdam, Unmixed 2-dimensional local domains . .

D. B. McAlister and Norman R. Reilly, E-unitary covers for inverse semigroups...

William H. Meeks, III and Julie Patrusky, Representing codimension-one

homology classes by embedded submanifolds . . .

Premalata Mohapatro, Generalised quasi-Nörlund summability . .

Takahiko Nakazi, Superalgebras of weak-*Dirichlet algebras .

Catherine Louise Olsen, Norms of compact perturbations of operators .

William Henry Ruckle, Absolutely divergent series and isomorphism of

subspaces. II.

Bernard Russo, On the Hausdorff-Young theorem for integral operators .

Arthur Argyle Sagle and J. R. Schumi, Anti-commutative algebras and

homogeneous spaces with multiplications ............

Robert Evert Stong, Stiefel-Whitney classes of manifolds .

D. Suryanarayana, On a theorem of Apostol concerning Möbius functions of

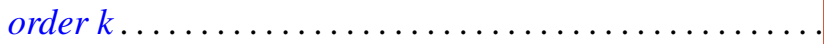

Yoshio Tanaka, On closedness of $C$ - and $C^{*}$-embeddings . . 